\title{
Sex-specific Effects of Localized Muscle Fatigue on Upper Body Kinematics During a Repetitive Pointing Task
}

Chen Yang ( $\sim$ chen.yang4@mail.mcgill.ca )

McGill University

Julie N. Côté

Jewish Rehabilitation Hospital

\section{Research Article}

Keywords: Fatigue, sex differences, kinematics, spine, variability

Posted Date: October 5th, 2021

DOl: https://doi.org/10.21203/rs.3.rs-944845/v1

License: (c) (1) This work is licensed under a Creative Commons Attribution 4.0 International License.

Read Full License 


\section{Abstract}

\section{Background}

Females are reported to have a higher risk of musculoskeletal disorders than males. Among risk factors for musculoskeletal disorders, the mechanism of muscle fatigue remains unclear. Especially how females and males adapt to localized fatigue is poorly understood. The purpose of the study was to examine the sex-specific effects of fatigue location on shoulder, elbow and spinal joint angles, and angular variabilities during a repetitive pointing task.

\section{Methods}

Seven males and ten females performed a standing repetitive pointing task when they were non-fatigued (NF), elbow-fatigued (EF), shoulder-fatigued (SF) and trunk-fatigued (TF), while trunk and upper body tridimensional kinematic data was recorded. Joint angles and angular variabilities of shoulder, elbow, upper thorax, lower thorax, and lumbar were calculated.

\section{Results}

Results showed that shoulder angles changed the most after EF in males, but after SF in females. The similarities between sexes were that SF increased the variabilities at upper (lateral flexion: $0.15^{\circ}$ greater than NF, rotation: $0.26^{\circ}$ greater than all other conditions) and lower thorax (lateral flexion: $0.13^{\circ}$ greater than NF, rotation: averagely $0.1^{\circ}$ greater than all other condition) in both sexes. TF altered upper thorax variability $\left(0.36^{\circ}\right.$ smaller than SF), lower thorax angle (lateral flexion: $3.00^{\circ}$ greater than NF, rotation: $1.68^{\circ}$ greater than SF), and lumbar angle (averagely $1.8^{\circ}$ smaller than all other conditions) in both sexes. However, females had greater lower thorax angle (lateral flexion: $8.3^{\circ}$ greater, $p=0.005$ ) as well as greater upper (rotation: $0.53^{\circ}$ greater, $p=0.006$ ) and lower thorax (rotation: $0.5^{\circ}$ greater, $p=0.007$; flexion: $0.6^{\circ}$ greater, $\mathrm{p}=0.014$ ) angular variabilities.

\section{Conclusions}

The overall greater lower and upper thorax angular variabilities suggested a more unstable spinal movement pattern in females. The kinematic differences between sexes highlighted a few sex differences in adapting the localized muscle fatigue.

\section{Background}

Work-related musculoskeletal disorders (WMSDs) are part of the inflammatory and degenerative conditions that are caused or exacerbated by occupational work. As a group of diseases which cause the most work absent and disability in Canada, it is well known that WMSDs have brought a huge economic burden and work time loss [1]. The risk factors of WMSDs need to be better understood to better protect workers. Studies have shown associations between muscle fatigue and WMSDs [2, 3]. Muscle fatigue can be induced by repetitive upper limb movement in various occupations. It is characterized by 
increased perceived effort and decreased maximal voluntary muscle force, velocity of muscle contraction and relaxation, and power output, among other findings [4-6]. The effects of muscle fatigue can be quantified using quantitative approaches such as those that help quantify muscle electromyography (EMG) changes, body posture adjustments as well as inter-joint coordination and motor variability change [7-9].

Using these approaches, Fuller et al. [8] found that body posture and shoulder kinematics were modified after fatigue in a standing pointing task. The movement-to-movement variabilities of shoulder and elbow motion amplitude were also found to increase after fatigue [10]. These studies also showed that muscle fatigue induced by the pointing task led to postural changes at other parts of the body such as trunk and elbow. Besides the postural changes, studies have also documented coordination adjustments with fatigue $[9,11,12]$. Previous studies showed that the human body adopted a different coordination pattern to compensate for muscle fatigue, where the central nervous system modified kinematics at other degrees of freedom when muscle fatigue was induced at a segment of the multi-segmental chain.

Other than fatigue, one's sex is another risk factor that contributes notably to WMSDs [13,14]. Studies have found that women have higher risks of upper body work-related pain and WMSDs $[13,14]$. This has been believed to be a result of different anthropometry, strength, flexibility, and other factors of biological origin [15-17]. In addition, previous studies have shown that females use different biomechanical techniques compared to males; for instance, upper body posture between females and males showed differences in multiple tasks. Females demonstrated higher upper body muscle activity than males in a painting task [18]. Straker et al. [19] observed that females had a more upright habitual sitting posture while using a computer. In a manual dexterity task, women had higher upper trapezius and anterior deltoid muscle activation amplitudes and functional connectivity between neighboring upper limb muscles compared to men, regardless of the fatigue state [20]. Moreover, during occupational tasks, some authors have pointed out that females have different trunk and spinal kinematics [19, 21]. Plamondon et al. [21] discovered that the lumbar spine of females was close to full flexion when initiating a lifting movement, which might increase risk of back injuries. Another study revealed that females exhibited greater anterior pelvic tilt during computer work [19]. Accordingly, the anthropometry and flexibility differences in the spines of females and males may play a role in affecting spinal kinematics [22].

When it comes to muscle fatigue, in general, females are usually less fatigable than males for similar relative intensity of isometric fatiguing contractions [23-25]. However, this has been shown to depend on the specific task [24]. The underlying physiological mechanism is thought to include sex differences in muscle mass, strength, blood flow perfusion, and fiber type proportion [26-29]. Interestingly, studies have found that females and males adopt different movement patterns when muscle fatigue arises. The varied fatigue adaptation might help explain the WMSDs risk sex difference. In a fatiguing upper limb task, the increase of trapezius muscle activation variability was found to be bigger in males than in females. Besides, the biceps activation variability decreased in males while it increased in females [30]. In a kinematic study, Bouffard et al. [31] showed that males decreased their humerothoracic elevation angle 
more while females increased humerothoracic elevation variability after fatigue in a standing pointing task. However, very few other studies focused on the sex-specific kinematic adaptation to muscle fatigue in dynamic tasks. With more studies, it may be possible to draw general conclusions that could help determine whether these sex differences in fatigue adaptations may help explain sex differences in mechanisms of WMSDs.

In real-life situations, fatigue may be induced globally by the repetition of the same multijoint task, or more locally at different joints, for instance when performing series of different occupational tasks that fatigue different body regions throughout a workday. Most of the previous studies have only focused on fatigue around one segment of the body and only few studies have investigated the difference when different joints of the body were fatigued. Cowley and Gates [32] found that distal and proximal muscle fatigue brought different kinematic changes to the body during a standing wrenching task. Proximal fatigue elicited greater trunk leaning angle and elbow flexion angle, while distal fatigue caused earlier wrist extension and increased wrench velocity. Yang et al. (2019) [33] compared the kinematic adaptations to localized fatigue at shoulder, elbow and trunk during a standing repetitive pointing task. It was shown that shoulder fatigue brought the biggest overall kinematic change, and that trunk fatigue induced adjustments of trunk-shoulder coordination. In comparison, localized fatigue at the elbow led to changes at shoulder and trunk but no changes of coordination. However, to our knowledge, no previous study has compared how men and women may differ in these adaptations to localized muscle fatigue when performing a multijoint task.

Therefore, the purpose of this study was to investigate the sex-specific kinematic adaptations to localized muscle fatigue during a standing repetitive pointing task when muscle fatigue was induced either at shoulder, elbow, or trunk. We expected to see sex differences after shoulder fatigue that were similar to those of Bouffard et al. [31], where males had a smaller shoulder elevation angle and females had a greater shoulder elevation variability. We also expected to see sex differences after elbow fatigue, since females have weaker upper limb strength, but less sex differences after trunk fatigue, since there are less sex differences in trunk strength [34-36].

\section{Methods}

\section{Participants}

Seventeen right-handed healthy young adults ( 7 men, 10 women; age $=23 \pm 2.7$ years; height $=172.9 \pm$ $8.8 \mathrm{~cm}$; body mass $=64 \pm 10.2 \mathrm{~kg}$ ) were recruited to participate in this study. The exclusion criteria were any previous experience in manual material handling work or any lower back pain, upper body injuries, musculoskeletal or cardiovascular impairment in the last 6 months before the data collection. All participants provided written informed consent prior to participation. The study was approved by the Research Ethics Board of the Centre for Interdisciplinary Research in Rehabilitation (CRIR) of Greater Montreal, and conducted in accordance with The Helsinki Declaration. 


\section{Protocol}

Participants performed the modified repetitive pointing tasks (RPT) before and after localized muscle fatigue was induced, as described in Yang et al. [33]. Briefly, the RPT requires that the participant, while comfortably standing, maintain the dominant arm horizontal, and move the index finger repetitively between a target placed at $30 \%$, and another one placed at $100 \%$ of arm reach [8]. The participant performed the RPTs 4 times: when they were not fatigued, after the lower back muscles were fatigued, after the dominant shoulder was fatigued, and after the dominant elbow was fatigued [33]. After the instruction and practice of the RPT, the participant had 10 minutes of rest, after which they performed the RPT for 30 seconds as the non-fatigued RPT (NFRPT). The Rating of Perceived Exertion (RPE) of the shoulder, elbow, and lower back muscles were asked using the Borg CR-10 scale before and after the NFRPT [37]. Then, series of fatiguing tasks were performed to induce muscle fatigue at shoulder, elbow and trunk one by one, using isometric efforts performed until exhaustion [33]. The order of the three fatiguing protocols was randomized, and right after each, the participant performed another $30 \mathrm{~s}$ of the RPT as the Fatigued RPT (SFRPT for shoulder fatigued RPT, EFRPT for elbow fatigued RPT, TFRPT for trunk fatigued RPT). The RPEs of shoulder, triceps brachii and lower back muscles were asked using Borg CR-10 scale before and after each fatigued RPT. Between each fatiguing task, the participant sat on a chair and passively recovered for at least 30 minutes [38]. The Borg CR-10 exertion score of the target muscle was asked every 5 minutes until it went back to the same number as the one before the NFRPT. More details of this protocol can be found in Yang et al. [33].

\section{Data acquisition}

A 7-camera motion capture system (MX3 VICON, Oxford Metrics Ltd., Oxford, UK) was used to record upper body kinematics (sampling frequency $=100 \mathrm{~Hz}$ ) during each RPT trial. Passive reflective markers were placed on the upper thorax (left and right T1, T6), lower thorax (left and right T8, T12), lumbar (left and right L1, S1), upper arm (acromioclavicular joint, lateral and medial epicondyle), forearm (lateral and medial epicondyle, styloid processes of ulna and radius), hand (styloid processes of ulna and radius, second and fifth metacarpophalangeal joint, index fingertip), pelvis (left and right anterior superior iliac spine, greater trochanter and S1), C7, Incisura Jugularis, and Xiphoid Process [39].

\section{Data analysis}

Kinematic data was low-pass filtered (digital 2th order Butterworth 199 filter, cut-off frequency $=7 \mathrm{~Hz}$, zero phase lag) in Visual 3D (C Motion, Germantown, MA). The segments of pelvis, trunk, right humerus, right forearm were built in Visual 3D to calculate the angles of trunk, right shoulder, and right elbow [39, 40]. According to the International Society of Biomechanics (ISB) recommendations, the shoulder angles were defined as followed: the first rotation $\left(Y^{\prime}\right)$ is the plane of elevation; the second rotation $(X)$ is the elevation; the third rotation $\left(Y^{\prime \prime}\right)$, internal/external rotation is the axial rotation angle. Shoulder and elbow 
kinematics were all calculated using Euler angles according to ISB recommendation. The segments of upper thorax, lower thorax, and lumbar were as described in Emery et al. [41]. The angles between upper thorax and lower thorax (UL), lower thorax and lumbar (LL), and lumbar and pelvis (LP) were calculated in Visual 3D. The angles were defined as follows: the first rotation was lateral flexion angle, second rotation was axial rotation angle, and third rotation was flexion/extension angle. For each of those joint angles, data from each forward movement cycle was first time-normalized to 101 data points. The average and standard deviation (SD) values of the 101 points were calculated for each movement cycle. Afterwards, the mean value of all the complete movement cycles (i.e., excluding data from the first and last 5 cycles to avoid accounting for incomplete cycles, and to avoid data boundary issues, i.e., cycles when the participant was accelerating to get into the rhythm, or decelerating to prepare to stop) was calculated to obtain the mean joint angle and mean SD values.

\section{Statistical analysis}

Generalized estimating equations (GEE) were used to examine the effects of Fatigue Location (NFRPT, SFRPT, EFRPT, TFRPT) and Sex (Male, Female) on each kinematics variable (joint angles, angular variabilities). The GEE approach was selected because it has more power than repeated measures analyses of variance (RM-ANOVA), it is less restrictive in its assumptions than RM-ANOVA, it helps estimate the average change per group, and it is robust against a misidentified choice of correlation matrix $[42,43]$. The LSD tests were used to apply the pairwise comparisons of Sex and Fatigue Location. Benjamini-Hochberg procedures were applied to correct the $p$ values and minimize type I error [44]. The false discovery rate was set at $5 \%$. Statistics were performed in Excel (Microsoft ${ }^{\circledR}$ Excel for Windows Version 15.26, Microsoft., US) and SPSS (SPSS Statistics v24, IBM Corp., US).

\section{Results}

\section{Endurance time}

On average, participants performed $9.18 \pm 3.13,8.00 \pm 1.90$ and $8.88 \pm 3.28$ trials for elbow, shoulder and trunk fatigue, respectively. Specifically, for shoulder, elbow and trunk fatiguing protocol, females performed $7.80 \pm 1.75,9.00 \pm 3.46$ and $8.70 \pm 3.92$ trials, and males performed $8.29 \pm 2.21,9.43 \pm 2.82$ and $9.14 \pm 2.34$ trials. There was no difference of endurance time between three fatiguing tasks (shoulder, elbow, and trunk; $p<0.0001)$ and between females and males $(p<0.0001)$ in any of those tasks.

\section{Shoulder angles:}

For the shoulder elevation angle, there was a significant interaction effect $(p=0.032)$ between fatigue location and sex. In males, the shoulder elevation angle was the smallest after $E F$ than any other condition (NF: $p<0.0001 ; S F: p=0.001 ; T F: p=0.027$ ). However, in females, the shoulder elevation angle was the smallest after SF compared to any other condition (NF: $p<0.0001 ; E F: p<0.0001 ; T F: p<0.0001$ ). 
As for other shoulder angles, there were no sex*fatigue location interaction or sex main effect. However, there was a significant fatigue location effect on the plane of elevation angle. The plane of elevation angle after SF was the smallest than any other conditions (NF: $p=0.011$; EF: $p<0.0001$; TF: $p<0.0001$ ). This implied that the humerus was less forward after the SF. Finally, there were no significant effects on shoulder angle variability.

\section{Elbow angles:}

No sex*fatigue location interaction or sex main effects were detected on the elbow angles. The elbow flexion/extension angle was the greatest after SF compared to any other conditions ( $2.61^{\circ}$ greater than after $\mathrm{NF}, \mathrm{p}=0.001 ; 3.30^{\circ}$ greater than after $\mathrm{EF}, \mathrm{p}=0.001$; and $3.68^{\circ}$ greater than after $\left.\mathrm{TF}, \mathrm{p}<0.0001\right)$. This indicated that the elbow was more flexed after SF. As for the variability, males had greater elbow flexion/extension variability than females $\left(4.07^{\circ}\right.$ greater, $\left.\mathrm{p}=0.001\right)$. In addition, the elbow flexion angle variability was greater after TF compared to NF $\left(1.32^{\circ}\right.$ greater, $\left.\mathrm{p}=0.002\right)$ and after $\mathrm{SF}\left(1.38^{\circ}\right.$ greater, $\mathrm{p}<0.01)$.

\section{Spinal angles:}

\section{Upper thorax (UL) angles:}

There was a significant interaction $(\mathrm{p}<0.0001)$ between sex and fatigue location on UL lateral flexion angle. In males, the UL lateral flexion angle was the smallest after SF compared to all other conditions ( $4.01^{\circ}$ smaller than $N F, p<0.0001 ; 3.75^{\circ}$ smaller than $E F, p<0.0001 ; 3.21^{\circ}$ smaller than $\left.T F, p<0.0001\right)$. In females however, the UL lateral flexion angle after SF was only smaller than NF ( $1.29^{\circ}$ smaller, $\left.p=0.02\right)$. This indicated that the upper thorax was leaning towards the non-reaching side the least after SF in males, but for females, the leaning after SF was only less than NF. The interaction effect between sex and fatigue location $(p<0.0001)$ also existed on UL rotation angle. In males, the UL rotation angle was the greatest after $S F$ compared to any other condition $\left(4.17^{\circ}\right.$ greater than $N F, p<0.0001 ; 3.58^{\circ}$ greater than $E F$, $p<0.0001 ; 5.06^{\circ}$ greater than TF, $\left.p<0.0001\right)$. In females, the UL rotation angle was not significantly affected by fatigue location. This implied that males rotated the upper thorax right more after SF and females remained the same. There was also an interaction effect between sex and fatigue location $(p=0.009)$ on UL flexion angle. In males, the UL flexion angle was same in all conditions. While in females, the UL flexion angle was the smallest after $\mathrm{EF}$ than any other conditions $\left(1.77^{\circ}\right.$ smaller than $\mathrm{NF}, \mathrm{p}=0.002$; $1.32^{\circ}$ smaller than $S F, p=0.015 ; 2.65^{\circ}$ smaller than $T F, p=0.001$ ). This indicated that females extended the UL less after EF. As for the variabilities, the UL lateral flexion variability was greater after SF than NF $\left(0.15^{\circ}\right.$ greater, $\left.p=0.04\right)$. The UL rotation variability after $S F$ was greater than all conditions $\left(0.26^{\circ}\right.$ greater than $\mathrm{NF}, \mathrm{p}=0.013 ; 0.17^{\circ}$ greater than $\mathrm{EF}, \mathrm{p}=0.024 ; 0.36^{\circ}$ greater than $\left.\mathrm{TF}, \mathrm{p}<0.0001\right)$. Besides, females had greater UL rotation variability $\left(0.53^{\circ}\right.$ greater, $\left.p=0.006\right)$ and smaller UL flexion variability $\left(0.44^{\circ}\right.$ smaller, $\mathrm{p}=0.038$ ) than males. 


\section{Lower thorax (LL) angles:}

There was no significant interaction effect between sex and fatigue on LL angles. However, females had significantly greater mean LL lateral flexion angle than males $\left(8.3^{\circ}\right.$ greater, $\left.p=0.005\right)$. Moreover, there was a significant fatigue location effect $(p<0.0001)$ on the $L L$ lateral flexion angle. The $L L$ lateral flexion angle after SF was greater than EF $\left(2.3^{\circ}, p=0.0002\right)$ and TF $\left(2.8^{\circ}, p<0.0001\right)$. It was also greater in NF than in EF $\left(2.5^{\circ}, p=0.006\right)$ and $\operatorname{TF}\left(3.0^{\circ}, p<0.0001\right)$. This indicated that after EF and TF, the lower thorax was leaning more towards the non-reaching side compared to NF. The LL rotation angle was greater after TF than SF $\left(1.7^{\circ}, p=0.007\right)$. As for the variabilities, there was an interaction effect between sex and fatigue location on LL lateral flexion variability. In males, there was no significant fatigue location effect. In females however, the LL lateral flexion variability in NF was smaller than it was after $\mathrm{SF}\left(0.2^{\circ}\right.$ smaller, $\left.p=0.0005\right)$ and $E F\left(0.2^{\circ}\right.$ smaller, $\left.p=0.0004\right)$. Besides, the $L L$ rotation $\left(0.5^{\circ}\right.$ greater in females, $\left.p=0.007\right)$ and flexion $\left(0.6^{\circ}\right.$ greater in females, $\left.p=0.014\right)$ variabilities were greater in females than they were in males.

\section{Lumbar (LP) angles:}

No significant interaction between sex and fatigue location was detected. As for the fatigue location effect, the only significant joint angle change was the LP lateral flexion angle, which was smaller after TF than all other conditions $\left(1.1^{\circ}\right.$ smaller than $\mathrm{NF}, \mathrm{p}=0.02 ; 2.0^{\circ}$ smaller than $\mathrm{EF}, \mathrm{p}<0.0001 ; 2.5^{\circ}$ smaller than $\mathrm{SF}, \mathrm{p}<0.0001)$. This implied that the lumbar was leaning the lumbar towards the non-reaching side the least after the TF. In addition, there was a significant sex difference on the LP lateral flexion angle. Males had greater LP lateral flexion angle than females $\left(5.4^{\circ}\right.$ greater, $\left.p=0.032\right)$. This indicated that males leaned their lumbar region more towards the non-reaching side than the females. As for angular variabilities, there were interaction effects between sex and fatigue location on LP lateral flexion angle variability $(p=0.001)$ and LP rotation angle variability $(p<0.0001)$. In males, the LP lateral flexion variability was greater after $S F$ than after EF ( $p=0.02)$ and TF $(p=0.02)$, whereas in females, it was smaller after $S F$ than after $E F(p=0.025)$ and TF $(p=0.025)$. Besides, the LP rotation variability in males was greater after SF than after $E F(p=0.003)$. But in females, it remained the same in all fatigue location conditions.

\section{Discussion}

This study assessed whether there are sex differences in the effects of localized muscle fatigue on upper body kinematics during a repetitive upper limb task. During the fatiguing protocols, the resistance was controlled so that the male and female participants reached the same perceived fatigue level in a similar amount of time. Our results show that despite some similarities between sexes, females and males showed some differences in how localized fatigue affected how they accomplished the repetitive pointing task, especially in the spinal joint angles. More specifically, in agreement with our hypotheses, we showed sex differences after elbow fatigue with females having greater lower thorax rotation angle than males after elbow fatigue. Besides, males changed trunk and shoulder angles the most after elbow 
fatigue. However, contrary to our hypotheses, we showed that males had greater shoulder elevation than females after shoulder fatigue.

\section{Interaction effects of sex and fatigue location}

Sex and fatigue location had interaction effects on shoulder and upper thorax angles as well as spinal angular variabilities. For shoulder angles, the present study revealed that males were mostly affected by $\mathrm{EF}$, while females were mostly affected by SF, compared to other fatigue locations. Our results showed that after EF, males dropped their humerus the most, while females had similar shoulder adaptations after SF. The kinematics difference in specific localized fatigue between sexes might be explained by several reasons. First, there are known sex differences in fatigability at different muscles. Multiple studies have shown that females are less fatigable than males in the majority of isometric fatiguing tasks [4547]. This could explain why, in the current study, females may have experienced more kinematic changes by the end of the shoulder fatiguing task even though the perceived fatigue level was the same as for males. More specifically, females had greater kinematic adaptations after SF compared with EF and TF. However, specifically at elbow, Hunter revealed that males had greater EMG amplitude increases of elbow flexors in an isometric elbow fatiguing task [23]. This could help explain our findings that males might be more fatigued after the elbow fatiguing protocol, leading to greater kinematic adaptations compared to SF and TF. Indeed, sex differences in fatigability are task-specific and can be affected by many factors. According to the literature review by Hunter [48], there is still a lack of understanding of sex differences in muscle fatigability. Second, there are sex differences in kinematic adaptations to the same localized fatigue. Studies have shown that females and males react to muscle fatigue differently, using different fatigue adaptations. For example, Srinivasan et al. [30] observed that males had greater trapezius EMG variability than females during the performance of the fatigued RPT. Similar findings can be found in Bouffard et al. [31], where authors detected greater humerothoracic angle decreases in males than females in the same task. In our study, results suggest that females and males adapted to the same localized fatigue differently. Especially for spinal angles, at the upper thorax, males changed the upper thorax angle the most at SF compared to all other fatigue locations. This is opposite compared to the trunk and shoulder angles, where females and not males reacted the most to SF. Besides, in terms of joint angular variabilities, only females increased lower thorax variability after SF and EF, and only males increased lumbar variability after SF. The sex*fatigue location interaction results implied the presence of sex differences in kinematics when adapting to a specific fatigue location. Females and males might adapt to localized fatigue (e.g. SF) by utilizing different motor pattern and altering different body parts.

\section{Sex difference regardless of the fatigue location}

Some kinematic differences between males and females were detected regardless of the fatigue location. Regarding the spinal angles, the lower thorax and lumbar lateral flexion angle were greater in females than males. For upper thorax lateral flexion angle, it was generally greater in males than in females. This 
might be a result of anthropometrical differences or due to the kinematic strategy that females and males adopted. In a study by Peharec et al. [16], the authors detected that pelvis range of motion is affected by sex, interpreted by female subjects having greater vertebral arcs than males in lateral flexion. Our results suggest that females tended to recruit the degrees of freedom at lower spine while males tended to recruit them at upper spine. In the study by Srinivasan et al. [30], the authors found that males showed greater trapezius variability than females after fatigue, which also supports our finding of males altering upper spinal kinematics. In terms of angular variabilities, we observed that males had greater elbow variabilities. However, for spinal angular variability, it was females who had greater variabilities at upper thorax, lower thorax, and lumbar region. The spinal angle variability reflected the variability of the sectional spine relative to the adjacent section. These sex differences suggest that females possessed more unstable spinal movement than males, while males showed more unstable arm movement than females during the RPT. In another study also using an RPT task, Bouffard et al. [31] observed a greater elbow flexion variability in females than males, which is contrary to our results. This might be explained by slight differences between the RPT tasks in these two studies. In the current study, the movement frequency was doubled and the participant was holding a weight in their moving hand while performing the RPT. Besides, the observed sex differences in variability occurred mostly in the frontal plane, which may be due to differences in lateral flexion flexibility or in frontal plane anthropometrics between the sexes. However, more studies are needed to further explain the spinal kinematic differences between the sexes when performing upper limb tasks.

\section{Fatigue location effect on spinal kinematics adaptation regardless of sex}

In the present study, we further separated the spine into upper thorax, lower thorax, and lumbar segment, and calculated the relative angles between spinal sections. The results showed that TF had the greatest overall impact on the spinal kinematic adaptations. It altered the lower thorax and lumbar angles as well as upper thorax angular variability. Previous studies using the RPT have shown the important role of the trunk in muscle fatigue adaptations [8, 9, 31]. Yang et al. [33] did not observe trunk angle changes after $\mathrm{TF}$, but in the present study, we showed that TF altered the lower thorax and lumbar angles. To our knowledge, this is the first study to show how localized trunk fatigue affects different spinal angles during a repetitive upper limb movement. Yang et al. [33] revealed that EF led to greater trunk lateral flexion angle. In our study, we further explained the EF effects on trunk angle where EF resulted in greater lower thorax lateral flexion angle. Our results suggest that distal fatigue can affect proximal joint kinematics in a multi-joint movement. Moreover, it also implied that the spine can be adjusted at different sections to compensate for different localised fatigue. As for SF, previous studies using the RPT have shown the effects of SF on joint angular variabilities [9, 10,30]. Bouffard et al. [31] detected increased trunk variabilities in three planes at the end of the RPT. Yang et al. [33] revealed that the trunk variabilities increased in two planes after SF. The present study showed that it was the variabilities of upper thorax and lower thorax, but not at the lumbar region, that increased. Since the lumbar segment is closer to the 
center of mass (CoM), this may suggest that SF impaired the upper spine but not the lower spine so that the CoM could be maintained. In the study by Fuller et al. [10], researchers found that CoM variability was preserved so that the task performance can be performed successfully, a finding that our current results seem to support.

\section{Limitations}

Even though some different kinematic changes were detected between females and males, the small sample size of this study cannot be neglected. Future studies with greater sample size might be needed. An inherent sex difference in how shoulder fatigue was induced may represent another limitation. One piece of weight $(0.7 \mathrm{~kg})$ was inserted to the female's wrist band while the male participant had two pieces $(1.4 \mathrm{~kg})$. The weight and fatiguing task intensity were not normalized according to the participants' muscle strength but were based on pilot studies, but with a goal of obtaining comparable endurance times in the different localized fatigue protocols, which we were able to achieve.

\section{Perspectives and significance}

The findings of this study showed similarities and differences between the sexes in how they adapt to fatigue. These results suggest that females and males might be placed at similar injury risk for some body parts but different risk level for other body parts. These results may have important consequences on jobs whose workforce may contain both males and females. For instance, since elbow fatigue had the greatest impact on males, but shoulder fatigue had the greatest impact on females, it may be necessary to allow more time between elbow efforts and multi-joint tasks for males, and more time between shoulder efforts and multi-joint tasks for females. Another strategy would be to adopt different work sequences in jobs that combine different tasks that lead to localized muscle fatigue for male and female employees. Future research may include larger amount of data collected in real workplaces to provide more ecologically information of sex differences in fatigue adaptations.

\section{Conclusion}

This study showed that females and males adapted to elbow and shoulder muscle fatigue differently. Males leaned and rotated the upper thorax to the non-reaching side when shoulder muscle was fatigued. Females adopted the same kinematic compensation pattern when the elbow muscle was fatigued. Females had greater upper thorax and lower thorax variability on multiple planes. Conversely, males had greater elbow flexion variability. Finally, spinal kinematics were altered differently to adapt to muscle fatigue at different body locations, regardless of sex. Future studies are needed to better understand the origin of the sex differences in kinematic adaptations to different muscle fatigue locations and estimate whether these differences may help explain the known sex differences in workplace injuries. 


\section{Abbreviations}

CoM: Center of Mass

CRIR: Centre for Interdisciplinary Research in Rehabilitation of Greater Montreal

EF: Elbow-Fatigued

EMG: Electromyography

FRQS: Fond de Recherche du Québec - Santé

GEE: Generalized Estimating Equations

ISB: International Society of Biomechanics

LL: Lower thorax - Lumbar

LP: Lumbar - Pelvis

NF: Non-Fatigued

RM-ANOVA: Repeated Measured Analysis of Variance

RPE: Rating of Perceived Exertion

RPT: Repetitive Pointing Task

SD: Standard Deviation

SF: Shoulder-Fatigued

TF: Trunk-Fatigued

WMSDs: Work-related musculoskeletal disorders

\section{Declarations}

\section{Availability of data and materials}

The datasets used and/or analyzed during the current study are available from the corresponding author on reasonable request.

\section{Acknowledgments}

We thank Ms. Kim Emery and Maxana Weiss for their help with the data collection. 


\section{Funding}

This study was supported by PhD fellowships from the Bloomberg Manulife Fellowship, Centre for Interdisciplinary Research in Rehabilitation of Great Montreal (CRIR) Doctoral Bursary Program, Canadian MSK Rehab Research Network Trainee Award, Fond de Recherche du Québec - Santé (FRQS) and operating grants from Natural Sciences and Engineering Council of Canada.

\section{Authors' contributions}

$\mathrm{CY}$ and $\mathrm{JC}$ concepted the research idea and designed the study. $\mathrm{CY}$ collected data, performed data analysis, interpreted the results and drafted the manuscript. JC supervised data collection and edited the manuscript.

\section{Ethics declarations}

Ethics approval and consent to participate

The protocol, including the content of recruitment flyers, was approved by the ethics committee of the Center for Interdisciplinary Research in Rehabilitation (CRIR) of Greater Montreal.

\section{Consent for publication}

Consent for each participant's data to be published was gained at the time of data collection.

\section{Competing interests}

No competing/conflicting interests were identified for either author or any of the funding agencies.

\section{References}

1. Statistics Canada, C.o.C., Canadian Community Health Survey (2015-2016). 2016, Ottawa, Canada.

2. Sommerich, C.M., J.D. McGlothlin, and W.S. Marras, Occupational risk factors associated with soft tissue disorders of the shoulder: a review of recent investigations in the literature. Ergonomics, 1993. 36(6): p. 697-717.

3. Côté, J.N., Adaptations to neck/shoulder fatigue and injuries, in Progress in motor control. 2014, Springer. p. 205-228.

4. Beelen, A. and A. Sargeant, Effect of fatigue on maximal power output at different contraction velocities in humans. Journal of Applied Physiology, 1991. 71(6): p. 2332-2337. 
5. Vollestad, N.K., Measurement of human muscle fatigue. J Neurosci Methods, 1997. 74(2): p. 21927.

6. Enoka, R.M. and D.G. Stuart, Neurobiology of muscle fatigue. Journal of applied physiology, 1992. 72(5): p. 1631-1648.

7. Bigland-Ritchie, B., et al., Contractile speed and EMG changes during fatigue of sustained maximal voluntary contractions. Journal of neurophysiology, 1983. 50(1): p. 313-324.

8. Fuller, J., et al., Posture-movement changes following repetitive motion-induced shoulder muscle fatigue. Journal of Electromyography and Kinesiology, 2009. 19(6): p. 1043-1052.

9. Yang, $\mathrm{C}$., et al., Changes in movement variability and task performance during a fatiguing repetitive pointing task. Journal of Biomechanics, 2018. 76: p. 212-219.

10. Fuller, J.R., J. Fung, and J.N. Côté, Time-dependent adaptations to posture and movement characteristics during the development of repetitive reaching induced fatigue. Experimental Brain Research, 2011. 211(1): p. 133-143.

11. Côté, J.N., et al., Movement reorganization to compensate for fatigue during sawing. Experimental brain research, 2002. 146(3): p. 394-398.

12. Forestier, N. and V. Nougier, The effects of muscular fatigue on the coordination of a multijoint movement in human. Neuroscience letters, 1998. 252(3): p. 187-190.

13. Sarquis, L.M., et al., Classification of neck/shoulder pain in epidemiological research: a comparison of personal and occupational characteristics, disability and prognosis among 12,195 workers from 18 countries. Pain, 2016. 157(5): p. 1028.

14. Côté, J.N., A critical review on physical factors and functional characteristics that may explain a sex/gender difference in work-related neck/shoulder disorders. Ergonomics, 2012. 55(2): p. 173-182.

15. Burgess, K.E., P. Graham-Smith, and S.J. Pearson, Effect of acute tensile loading on gender-specific tendon structural and mechanical properties. Journal of orthopaedic research, 2009. 27(4): p. 510516.

16. Peharec, S., et al., Kinematic measurement of the lumbar spine and pelvis in the normal population. Collegium antropologicum, 2007. 31(4): p. 1039-1042.

17. Faber, A., K. Hansen, and H. Christensen, Muscle strength and aerobic capacity in a representative sample of employees with and without repetitive monotonous work. International archives of occupational and environmental health, 2006. 79(1): p. 33-41.

18. Rosati, P.M., J.N. Chopp, and C.R. Dickerson, Investigating shoulder muscle loading and exerted forces during wall painting tasks: influence of gender, work height and paint tool design. Applied ergonomics, 2014. 45(4): p. 1133-1139.

19. Straker, L.M., et al., Neck/shoulder pain, habitual spinal posture and computer use in adolescents: the importance of gender. Ergonomics, 2011. 54(6): p. 539-546.

20. Minn, S. and J.N. Côté, Gender differences in sensorimotor characteristics associated with the performance of a manual dexterity task at shoulder height. Journal of Electromyography and 
Kinesiology, 2018. 42: p. 143-150.

21. Plamondon, A., et al., Difference between male and female workers lifting the same relative load when palletizing boxes. Applied ergonomics, 2017. 60: p. 93-102.

22. Pries, E., et al., The effects of age and gender on the lumbopelvic rhythm in the sagittal plane in 309 subjects. Journal of biomechanics, 2015. 48(12): p. 3080-3087.

23. Hunter, S.K., et al., Fatigability of the elbow flexor muscles for a sustained submaximal contraction is similar in men and women matched for strength. Journal of Applied Physiology, 2004. 96(1): p. 195202.

24. Hunter, S.K., Sex differences and mechanisms of task-specific muscle fatigue. Exercise and sport sciences reviews, 2009. 37(3): p. 113.

25. Ditor, D.S. and A. Hicks, The effect of age and gender on the relative fatigability of the human adductor pollicis muscle. Canadian journal of physiology and pharmacology, 2000. 78(10): p. 781790.

26. Simoneau, J.-A. and C. Bouchard, Human variation in skeletal muscle fiber-type proportion and enzyme activities. American Journal of Physiology-Endocrinology And Metabolism, 1989. 257(4): p. E567-E572.

27. Staron, R.S., et al., Fiber type composition of the vastus lateralis muscle of young men and women. Journal of histochemistry \& cytochemistry, 2000. 48(5): p. 623-629.

28. Roepstorff, C., et al., Higher skeletal muscle a2AMPK activation and lower energy charge and fat oxidation in men than in women during submaximal exercise. The Journal of physiology, 2006. 574(1): p. 125-138.

29. Welle, S., R. Tawil, and C.A. Thornton, Sex-related differences in gene expression in human skeletal muscle. PloS one, 2008. 3(1): p. e1385.

30. Srinivasan, D., et al., Gender differences in fatigability and muscle activity responses to a short-cycle repetitive task. Eur J Appl Physiol, 2016. 116(11-12): p. 2357-2365.

31. Bouffard, J., et al., Sex differences in kinematic adaptations to muscle fatigue induced by repetitive upper limb movements. Biology of sex differences, 2018. 9(1): p. 17.

32. Cowley, J.C. and D.H. Gates, Proximal and distal muscle fatigue differentially affect movement coordination. PloS one, 2017. 12(2): p. e0172835.

33. Yang, C., S. Leitkam, and J.N. Côté, Effects of different fatigue locations on upper body kinematics and inter-joint coordination in a repetitive pointing task. PloS one, 2019. 14(12).

34. Heyward, V.H., S.M. Johannes-Ellis, and J.F. Romer, Gender differences in strength. Research quarterly for exercise and sport, 1986. 57(2): p. 154-159.

35. Keller, T.S. and A.L. Roy, Posture-dependent isometric trunk extension and flexion strength in normal male and female subjects. Clinical Spine Surgery, 2002. 15(4): p. 312-318.

36. Evans, K., K.M. Refshauge, and R. Adams, Trunk muscle endurance tests: reliability, and gender differences in athletes. Journal of Science and Medicine in Sport, 2007. 10(6): p. 447-455. 
37. Borg, G.A., Psychophysical bases of perceived exertion. Med sci sports exerc, 1982. 14(5): p. 377381.

38. Davidson, B.S., M.L. Madigan, and M.A. Nussbaum, Effects of lumbar extensor fatigue and fatigue rate on postural sway. European journal of applied physiology, 2004. 93(1-2): p. 183-189.

39. Gates, D.H., et al., Range of motion requirements for upper-limb activities of daily living. American Journal of Occupational Therapy, 2016. 70(1): p. 7001350010p1-7001350010p10.

40. Wu, G., et al., ISB recommendation on definitions of joint coordinate systems of various joints for the reporting of human joint motion-Part Il: shoulder, elbow, wrist and hand. Journal of biomechanics, 2005. 38(5): p. 981-992.

41. Emery, K., et al., The effects of a Pilates training program on arm-trunk posture and movement. Clinical Biomechanics, 2010. 25(2): p. 124-130.

42. Ma, Y., M. Mazumdar, and S.G. Memtsoudis, Beyond Repeated measures ANOVA: advanced statistical methods for the analysis of longitudinal data in anesthesia research. Regional anesthesia and pain medicine, 2012. 37(1): p. 99.

43. Naseri, P., et al., Comparison of generalized estimating equations (GEE), mixed effects models (MEM) and repeated measures ANOVA in analysis of menorrhagia data. Journal of Paramedical Sciences, 2016. 7(1): p. 32-40.

44. Benjamini, Y. and Y. Hochberg, Controlling the false discovery rate: a practical and powerful approach to multiple testing. Journal of the Royal statistical society: series B (Methodological), 1995. 57(1): p. 289-300.

45. Maughan, R., et al., Endurance capacity of untrained males and females in isometric and dynamic muscular contractions. European journal of applied physiology and occupational physiology, 1986. 55(4): p. 395-400.

46. Hunter, S.K. and R.M. Enoka, Sex differences in the fatigability of arm muscles depends on absolute force during isometric contractions. Journal of Applied Physiology, 2001. 91(6): p. 2686-2694.

47. Clark, B.C., et al., Gender differences in skeletal muscle fatigability are related to contraction type and EMG spectral compression. Journal of Applied Physiology, 2003. 94(6): p. 2263-2272.

48. Hunter, S.K., Sex differences in human fatigability: mechanisms and insight to physiological responses. Acta physiologica, 2014. 210(4): p. 768-789.

\section{Figures}



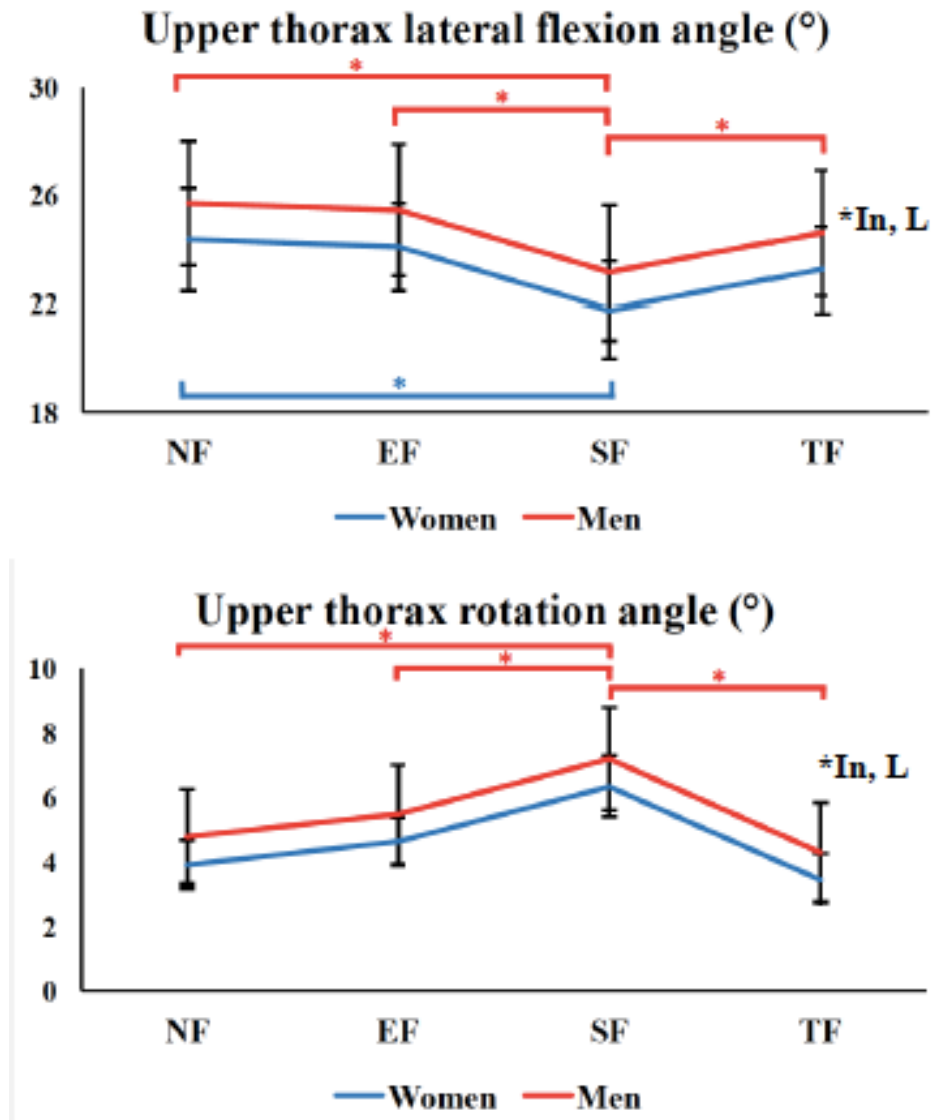

Upper thorax flexion angle $\left({ }^{\circ}\right)$

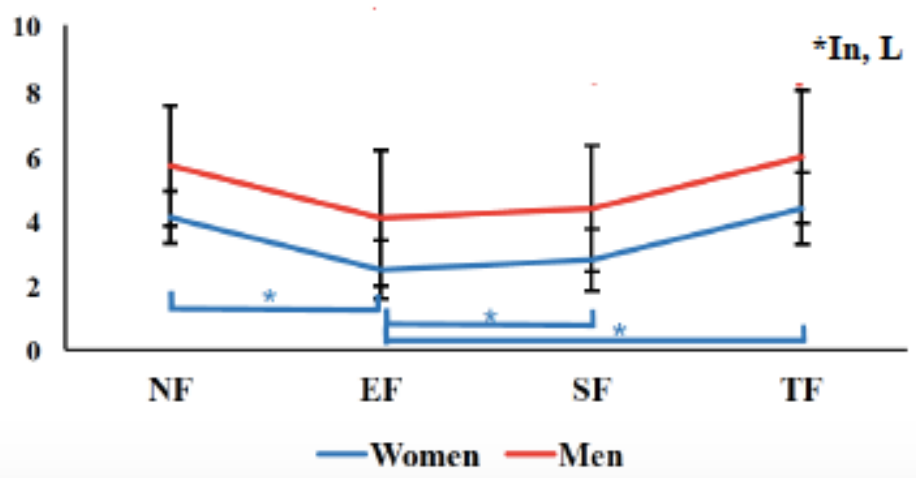

Upper thorax lateral flexion variability $\left({ }^{\circ}\right)$

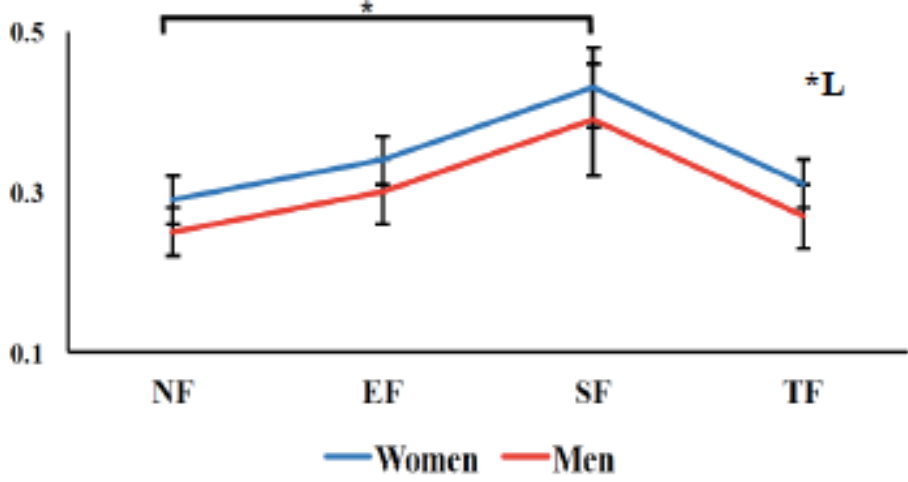

Upper thorax rotation variability $\left(^{\circ}\right)$

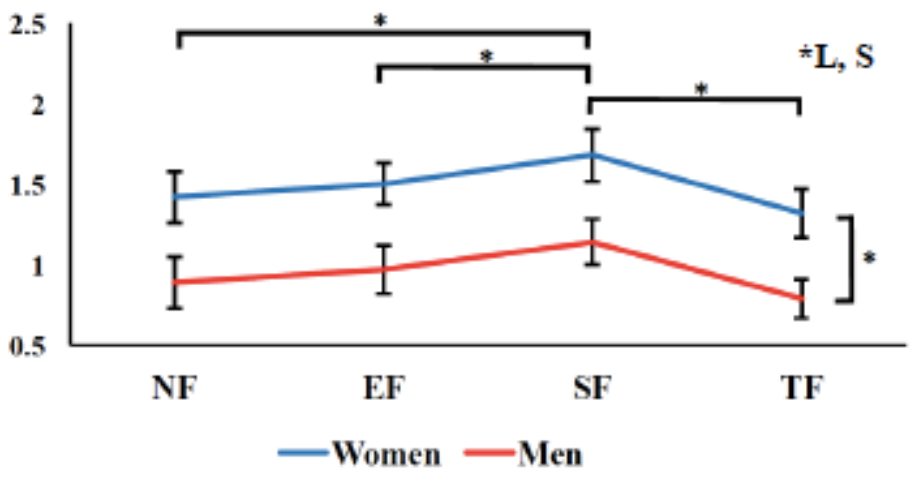

Upper thorax flexion variability $\left({ }^{\circ}\right)$

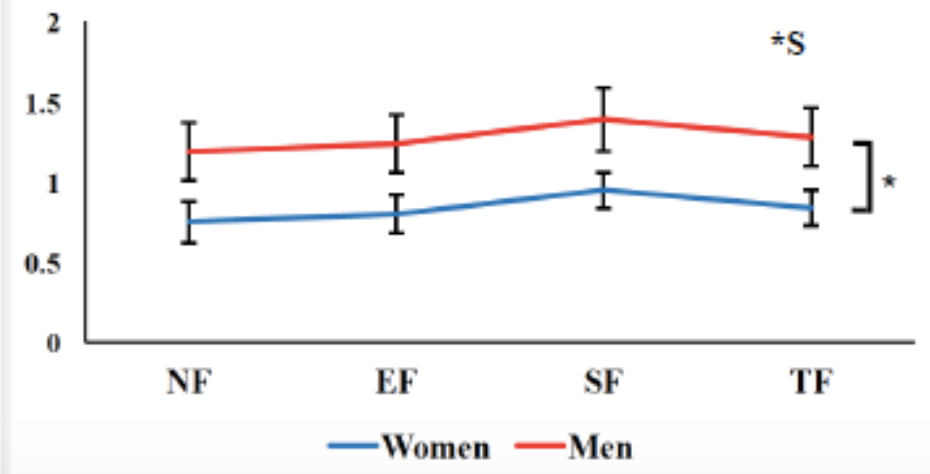

Figure 1

Upper thorax angles and angular variabilities. The blue lines indicate joint angles and angular variabilities in women, and the red lines indicate the joint angles and angular variabilities in men. The vertical bars above and below each point represent the standard deviation. The blue brackets and * indicate significant differences between location in women, the red brackets and * indicate significant differences between locations in men, and black brackets and * indicate significant differences between locations regardless of sex or the sex differences regardless of location. "S", "L", "In" stand for significant effects of sex, fatigue location, and interaction between sex and fatigue location, respectively. 


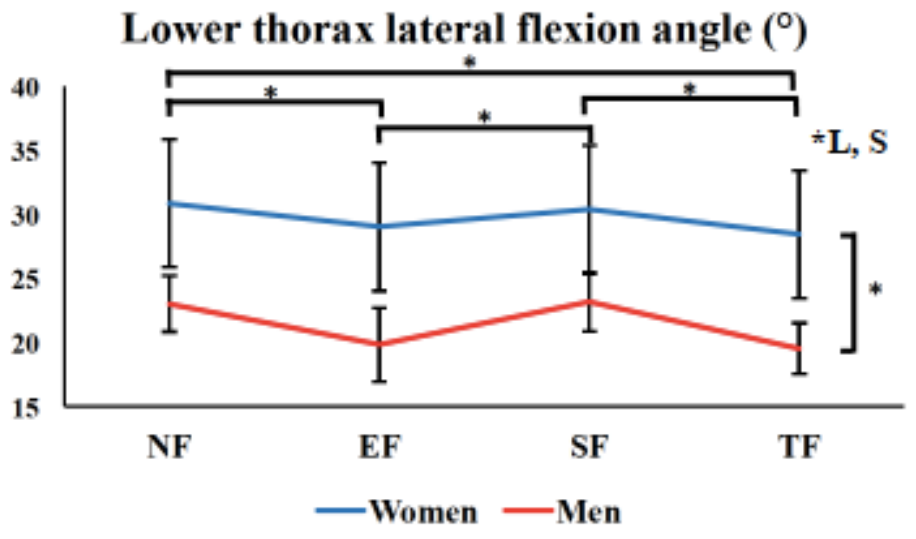

Lower thorax rotation angle $\left({ }^{\circ}\right)$

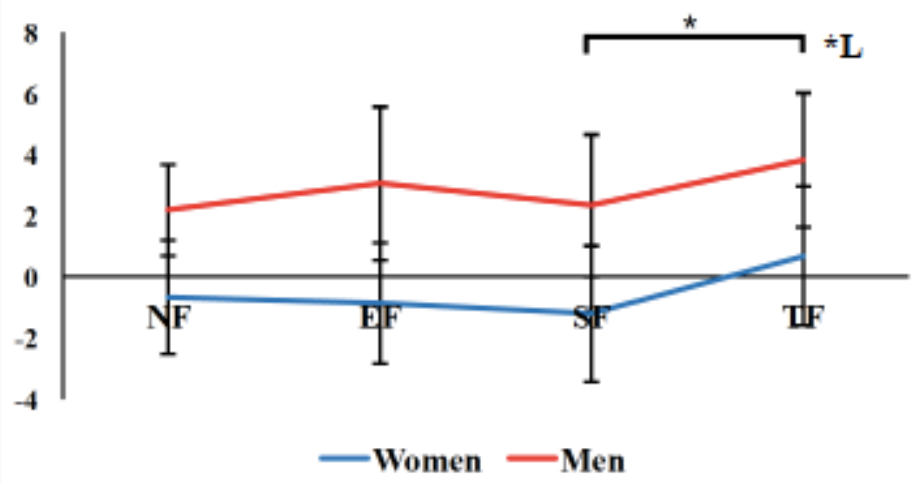

Lower thorax flexion angle $\left({ }^{\circ}\right)$

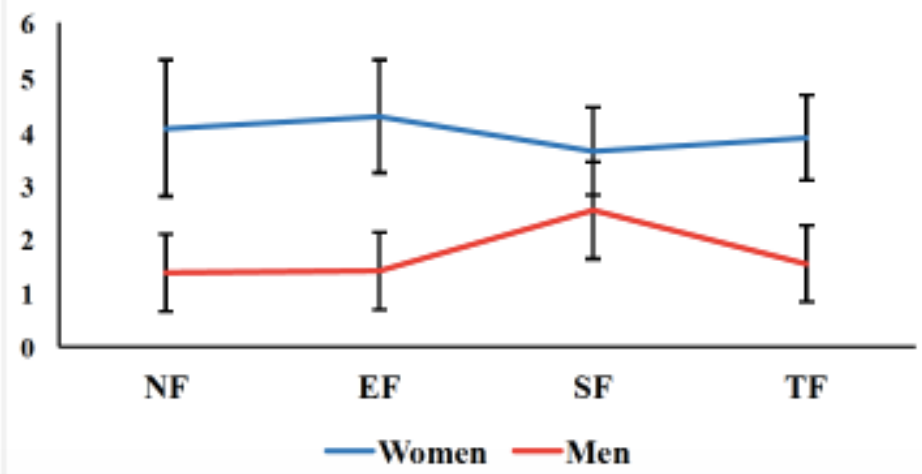

Lower thorax lateral flexion variability $\left({ }^{\circ}\right)$

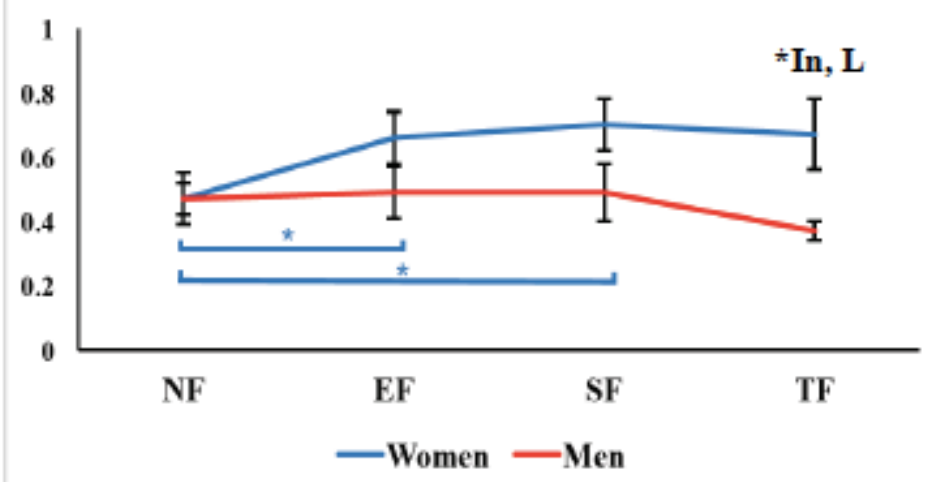

Lower thorax rotation variability $\left({ }^{\circ}\right)$

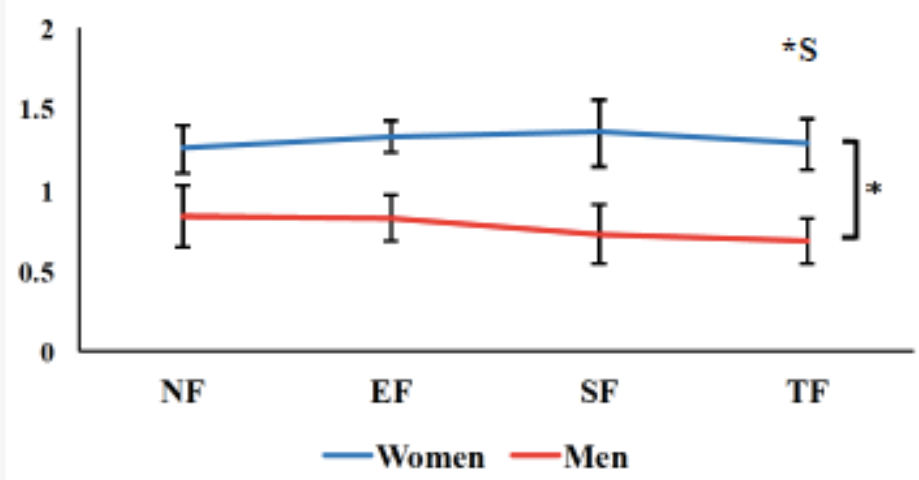

Lower thorax flexion variability $\left({ }^{\circ}\right)$

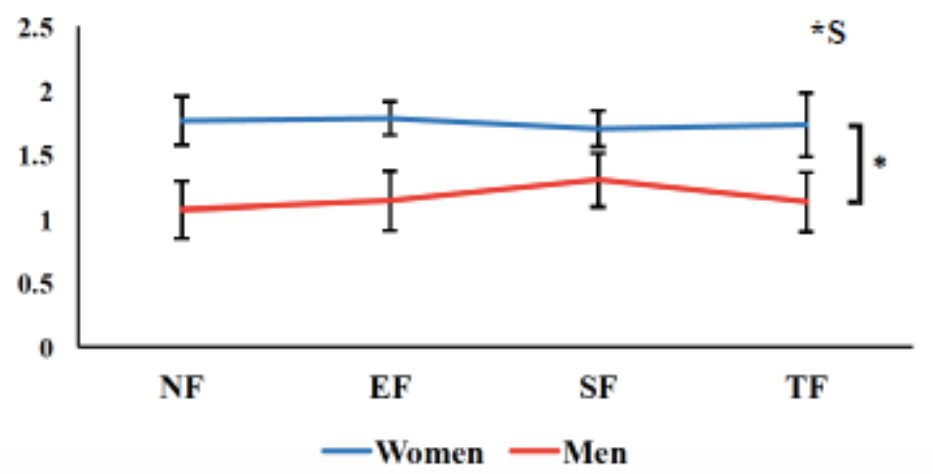

Figure 2

Lower thorax angles and angular variabilities. The blue lines indicate joint angles and angular variabilities in women, and the red lines indicate the joint angles and angular variabilities in men. The vertical bars above and below each point represent the standard deviation. The blue brackets and * indicate significant differences between location in women, the red brackets and * indicate significant differences between locations in men, and black brackets and * indicate significant differences between locations regardless of sex or the sex differences regardless of location. "S", "L", "In" stand for significant effects of sex, fatigue location, and interaction between sex and fatigue location, respectively. 


\section{Lumbar lateral flexion angle $\left({ }^{\circ}\right)$}

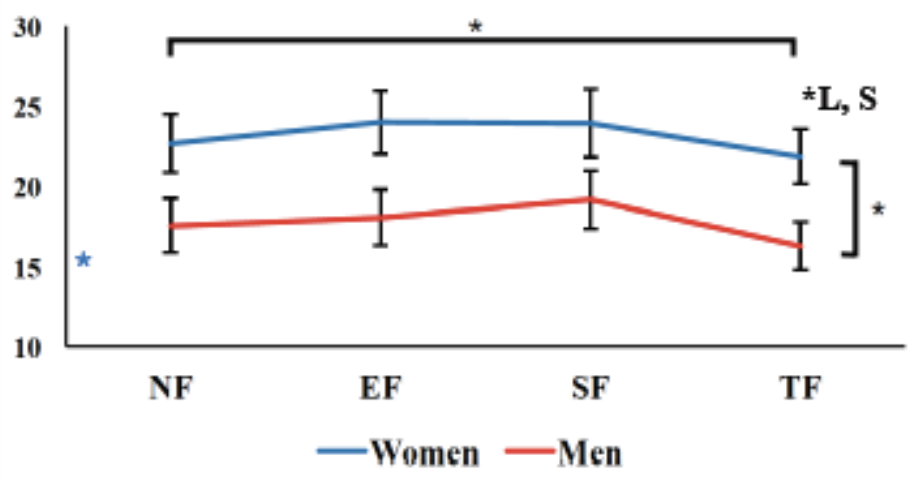

Lumbar rotation angle $\left({ }^{\circ}\right)$

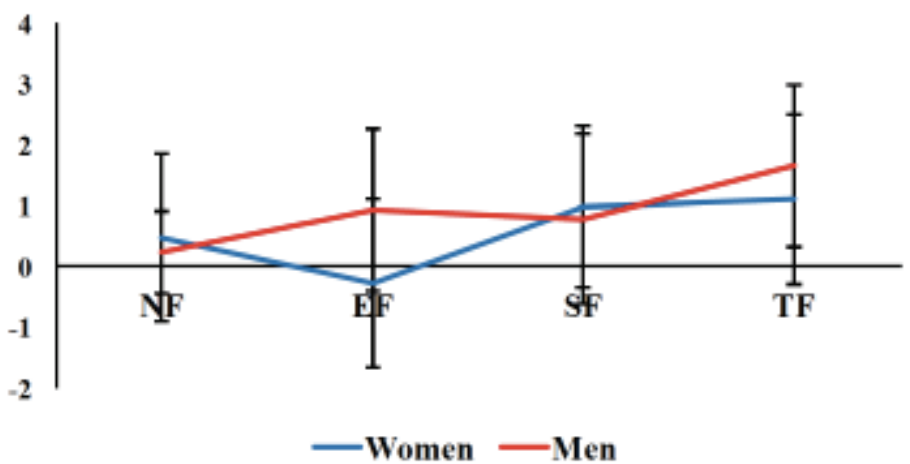

Lumbar flexion angle $\left({ }^{\circ}\right)$

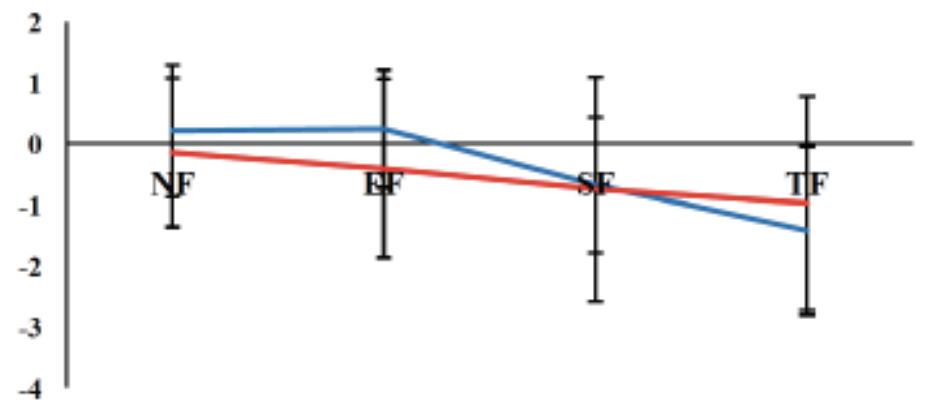

—Women -Men
Lumbar lateral flexion variability $\left({ }^{\circ}\right)$

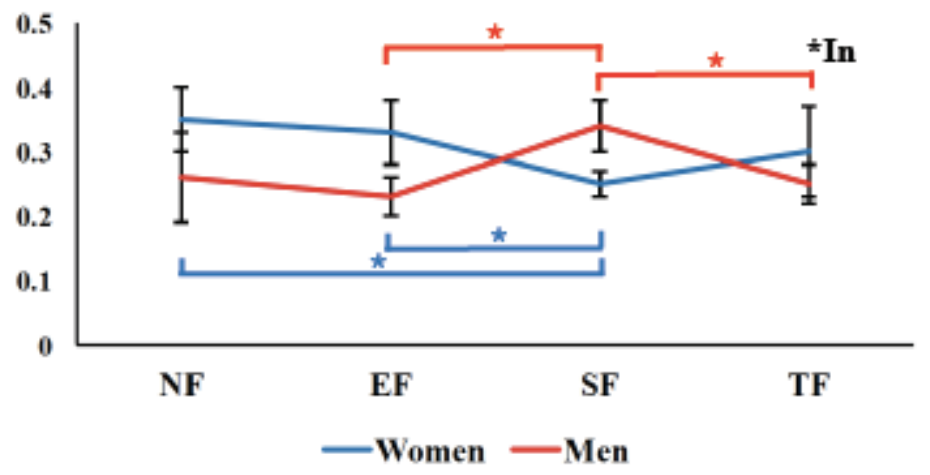

Lumbar rotation variability $\left({ }^{\circ}\right)$

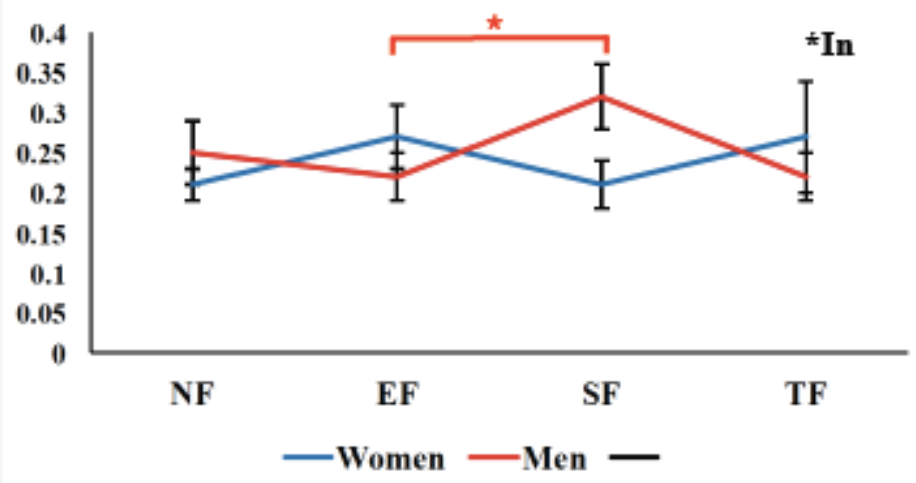

Lumbar flexion variability $\left({ }^{\circ}\right)$

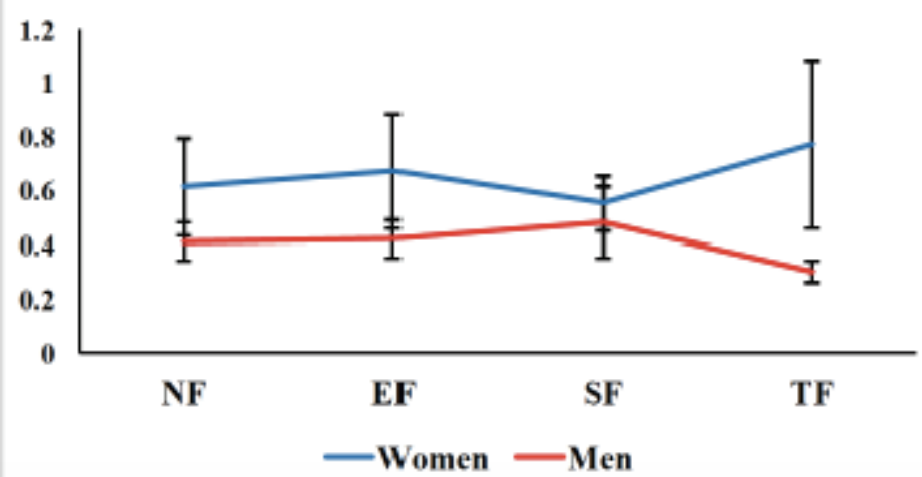

Figure 3

Lumbar angles and angular variabilities. The blue lines indicate joint angles and angular variabilities in women, and the red lines indicate the joint angles and angular variabilities in men. The vertical bars above and below each point represent the standard deviation. The blue brackets and * indicate significant differences between location in women, the red brackets and * indicate significant differences between locations in men, and black brackets and * indicate significant differences between locations regardless of sex or the sex differences regardless of location. "S", "L", "In" stand for significant effects of sex, fatigue location, and interaction between sex and fatigue location, respectively. 